\title{
Rapid Cortisol and Testosterone Responses to Sex-Linked Stressors: Implications for the Tend-and-Befriend Hypothesis
}

\author{
Jennifer Byrd-Craven ${ }^{1}$ • Jessica L. Calvi ${ }^{2}$ - Shelia M. Kennison ${ }^{1}$
}

Published online: 19 April 2016

(C) Springer International Publishing 2016

\begin{abstract}
Current evolutionary theories regarding the nature of hormonal responses to a variety of salient social stimuli are incomplete in yielding evidentiary support for their assertions. This study offers more nuanced evidence for the Tend-andBefriend model of sex differences in responses to social stimuli. Participants were randomly assigned to a mortality salience prime or a control condition prior to viewing a video of an out-group threat or a video of infants crying. Cortisol and testosterone responses were assessed. The results showed that in mortality salience conditions, females showed significantly higher cortisol responses to infants crying compared to males. Further, in both mortality salience and control conditions, females showed significantly higher testosterone responses to the crying infants than males. Males showed a greater testosterone response to viewing an out-group threat. Mortality salience prime did not impact testosterone responses in either sex. Results point to a more nuanced representation of hormonal responses to social stimuli and the need for multisystem measurement.
\end{abstract}

Keywords Sex differences · Tend-and-befriend · Cortisol . Testosterone $\cdot$ Mortality salience

This research was partially supported by a grant from the National Science Foundation (SMA 1358847).

Jennifer Byrd-Craven

jennifer.byrd.craven@okstate.edu

1 Department of Psychology, Oklahoma State University, 116 North Murray Hall, Stillwater, OK 74078, USA

2 University of Nebraska, Lincoln, NE, USA
Social challenges are inherently associated with a host of hormonal responses, presumably aimed at directing attention and other resources in such a way as to facilitate a behavioral response. Hormonal responses to social challenges have been shown to differ in some contexts between the sexes. For example, Taylor et al. (2000) outlined an evolutionary framework for predicting biobehavioral sex differences in responses to social challenge, termed "Tend-and-Befriend," and posited sex differences in responses to stress, which are based on sexually selected differences in emphases in social environments. Under this framework, males are expected to follow the classic fight-or-flight response pattern, while females are expected to follow a behavioral and physiological pattern that elicits care and protection of offspring when present (tending) and that involves the seeking of affiliation and social support, primarily from same-sex friends (befriending component). Thus, the tend-and-befriend response pattern is most likely to be evident under conditions that present cues involving the opportunity to provide care to altricial conspecifics and/ or seek affiliative support from others (Taylor 2006).

The "tending" predisposition to infant distress cues is expected to be regulated by the activity of the nervous system as well as other related hormonal systems aimed at facilitating behavioral responsiveness. This responsiveness would then be expected to regulate tending behaviors, particularly in humans as cooperative breeding species (Hrdy 2007). In fact, the hypothalamic-pituitary-adrenal (HPA) axis, as measured by cortisol, has been shown to be responsive to infant distress cues and associated with maternal behavior following childbirth (Fleming et al. 1997a, b; Stallings et al. 1997). Further, nulliparous women also show greater cortisol responses to an audio recording of infants crying compared to an audio recording of neutral verbal passages (Giardino et al. 2008).

A more fine-tuned model of men's hormonal responses to threats has been suggested by Geary and Flinn (2002), but few 
empirical investigations have attempted to test their assertions. The suggested modifications to the Tend-and-Befriend model assert that males tend and befriend as well under conditions of threat, but that the conditions and behaviors initiating these responses are expected to differ according to differential selection pressures. Briefly, this model emphasizes the importance of male philopatry through much of our evolutionary history, where men have historically operated within kinbased coalitions. Men are more likely to form or re-establish coalitions (befriending) under conditions of threat to the group as a way of protecting reproductive resources against other coalitions (tending) (Geary 2010; Savin-Williams 1979). An out-group threat is a cue that is expected to trigger hormonal activity as a part of a coordinated behavioral response to meet the challenge. Consistent with this, successes in competitions against outside members of a group are associated with testosterone elevations (Bernhardt et al. 1998; Gonzalez-Bono et al. 2000; Kivlighan et al. 2005; Oxford et al. 2010; Wagner et al. 2002). Further, pre-competition testosterone and cortisol levels are associated with strength of coalitionary ties (Flinn et al. 2012).

Compounding the complex suite of behaviors related to testosterone is the traditional "Challenge Hypothesis" in men (Wingfield et al. 1990), which dominates much of the research literature on testosterone. In brief, this theory, along with Tend-and-Befriend, dichotomizes higher testosterone levels into the category of "competition" and low testosterone levels into the category of "parenting behaviors." However, more recent theories of social behavior, specifically the Steroid/ Peptide Theory of Social Bonds (van Anders et al. 2011), address the issue of levels of testosterone in the context of competition, aggression, and nurturing behaviors.

Traditionally, lower testosterone levels were thought to be related to all parenting behaviors (e.g., Wingfield et al. 1990); however, several recent studies have shown higher testosterone levels in relation to parenting situations. While much of the research directly links lower testosterone levels to nurturing behaviors in males, recent literature indicates a much more complex relationship with parenting behaviors than previously understood (van Anders et al. 2011). For example, fathers who heard a crying infant stimulus showed a greater percentage increase in testosterone than fathers who did not (Fleming et al. 2002). In addition, men within 3 weeks of their own child being born exhibited a significant increase in testosterone in response to hearing an infant cry (Storey et al. 2000). Thus, it is possible for increases in testosterone to be associated with infant cues of distress, e.g., men's increases in testosterone when nurturing behaviors were not present in response to simulated infant cries (van Anders et al. 2012).

Conversely, there is a marked lack of research addressing the testosterone responses of females to many of these competitive and parenting/nurturing behaviors (see van Anders 2013 for an extensive discussion). When administered endogenous testosterone, young women who were exposed to infant cries showed significantly higher activation in the thalamocingulate circuit. They also reported the experience as more unpleasant and "emotional" than comparable volume and spectral sounds (Bos et al. 2010). The authors posit that the increase in testosterone is due to the hormonal cascade in which testosterone is integral to the synthesis of estradiol, a sex hormone that is related to many parenting behaviors (Wynne-Edwards and Reburn 2000).

Like the Geary and Flinn (2002) modifications to Tendand-Befriend model, the Steroid/Peptide Theory of Social Bonds (van Anders et al. 2011) applies a more multifaceted approach to the high/low dichotomy of testosterone in relation to human social behaviors. These theories point toward a need to address the complex hormonal cascades and the importance of context and perception in the laboratory studies addressing these complex social behaviors. How the hypothalamicpituitary-gonadal (HPG) axis is associated with such responses is not well understood. One purpose of the present research is to investigate if HPA and HPG responses are coordinated to a variety of social cues, namely out-group threats or cues of infant distress, as specified by the Tend-andBefriend hypothesis. We know little about how women, in particular, respond to cues outside of caretaking and social support responses. Recent research shows that the presence of same-sex rivals initiates a testosterone response in both men and women (Cobey et al. 2013; Maner and McNulty 2013; Miller et al. 2012).

Another salient aspect of the social environment is monitoring for potential threats to survival. Rival coalitions were a recurrent threat in our ancestral environment, and as such, we have evolved cognitive mechanisms associated with attention to and processing of this information (Kurzban et al. 2001). One useful approach to studying acute mortality threats is the Terror Management Theory (TMT). TMT focuses on the role that our understanding of our own inevitable mortality and associated existential anxiety plays in a broad range of cognitions and behaviors, including the need for self-esteem, identification with coalitions, and desire for close relationships (Greenberg et al. 1986; Solomon et al. 1991). The mortality salience (MS) hypothesis of TMT asserts that mortality reminders increase these needs, particularly related to affiliation with one's group (Wisman and Koole 2003). Sex differences in responses to MS have also been documented, with MS primes increasing accessibility of nationalistic (coalitional) constructs for men, but not for women, and MS increasing romantic accessibility for women, but not men (Arndt et al. 2002). Thus, mortality reminders may enhance biobehavioral responses in a sex-specific pattern.

Recent research investigated the biobehavioral responses to sex-specific stressors in the context of a mortality salience cue versus control context with no mortality salience cue (Byrd-Craven et al. 2015). Men or women 
were randomly assigned to either the mortality salience or control prime writing task and then to one of the two stressor conditions, which involved watching a video of a baby crying or a video of an out-group threat. The results showed that women's cortisol response was higher to the video of the crying baby than to the video of the out-group threat; the reverse pattern was found for men. Mortality salience affected responses of men only, as they showed a lower cortisol response after watching the video of the outgroup threat than in the control (i.e., no mortality salience) condition.

The current study tests the Tend-and-Befriend model, along with suggested revisions from Geary and Flinn (2002) that incorporate male tending and befriending under coalitional contexts. We tested conditions similar to those tested in Byrd-Craven et al. (2015) with comparable numbers of men and women, but assessed both cortisol and testosterone from all participants. Specific predictions are as follows: (1) We expected that males would be more sensitive to cues associated with out-group threat, in this case, aggressive for inflammatory speech directed at their coalition, by showing greater changes in cortisol compared to women and men in alternative conditions. (2) We also expected males experiencing a mortality salience prime to have enhanced physiological reactivity to these cues, particularly in the form of significant testosterone responses, consistent with previous research on biobehavioral responses to out-group threats (Oxford et al. 2010). (3) Females, on the other hand, were expected to be more sensitive to cues associated with caretaking, in this case, video recordings of babies crying. (4) Further, females experiencing a mortality salience prime were expected to have enhanced physiological reactivity to these cues, in the form of significant testosterone and cortisol responses.

\section{Method}

\section{Participants}

Participants were 155 undergraduates (76 males, M age $=19.48, \mathrm{SD}=2.35,69 \%$ European American), who were recruited from a pool of potential participants in exchange for course credit in their psychology courses. Participants were between 18 and 26 years, and were screened for factors known to potentially influence cortisol and testosterone levels (e.g., use of caffeine, hormonal contraceptives, medications, and illness).

\section{Measures}

Questionnaires Participants completed a battery of questionnaires as part of a larger study. Time was spent to complete questionnaires on demographics, personality, and thinking styles to habituate participants to the novel laboratory setting. Two post-task questions were given to participants after the second sample: (1) How much do you want to be around the other individuals around you? (2) How much do you want to be around close relationships (e.g., friends, family)? Questions were answered on a 1 ("I very much prefer to be alone") to 7 ("I very much prefer to be with a close friend/family member") scale.

Hormone Assays Saliva was obtained via passive drool in $2-\mathrm{mL}$ cryovials and stored at $-20{ }^{\circ} \mathrm{C}$. On the day of assay testing, saliva samples were centrifuged at $3000 \mathrm{rpm}$ for $15 \mathrm{~min}$ to remove mucins. Samples were assayed in duplicate for testosterone first, and then cortisol following manufacturer's instructions without modification (Salimetrics, State College, PA, USA). Testosterone levels are reported in picograms per milliliter, while cortisol levels are reported in micrograms per deciliter. The current study's testosterone inter-assay and intra-assay coefficients of variation were 9.64 and $6.21 \%$, respectively, and cortisol inter-assay and intra-assay coefficients of variation were 8.76 and $6.43 \%$, respectively.

\section{Procedure}

All procedures were reviewed and approved by the Institutional Review Board. The procedures followed Byrd-Craven et al. (2015). Participants were grouped into same-sex groups of 3-4 individuals. All participants participated between 1:00 and 5:00 p.m. to control for diurnal cortisol pattern. After consenting to participate in the study, participants answered self-report questionnaires. Participants were then randomly assigned to either a morality salience (MS) condition (experimental condition) or a dental pain prime (MS control condition) for $5 \mathrm{~min}$. Participants in the MS condition were told to "Please briefly describe the emotions that the thought of your own death arouses in you. Please describe, as specifically as you can, what you think will happen as physically die and once you are physically dead." Participants in the MS control condition were asked to do the same, but "death," "die," and "dead" were replaced with "experience dental pain." Next, participants were presented a 5-min video presentation with either the male-typical outgroup threat (Westboro Baptist Church footage of hate speech such as "God hates America," "Dead soldiers are God's wrath for your sins," and "your family is cursed.") or a femaletypical stressor of infants crying. Participants sat next to each other, equidistant from the monitor. Saliva samples were collected immediately before the mortality salience prime (T1), immediately after the sex-specific audio/visual presentation (T2), and $20 \mathrm{~min}$ after the end of the audio/visual presentation (T3) (See Fig. 1). During the 20-min waiting period, participants were asked to fill out a short series of questions 
Fig. 1 Timeline of experiment procedure and saliva sampling

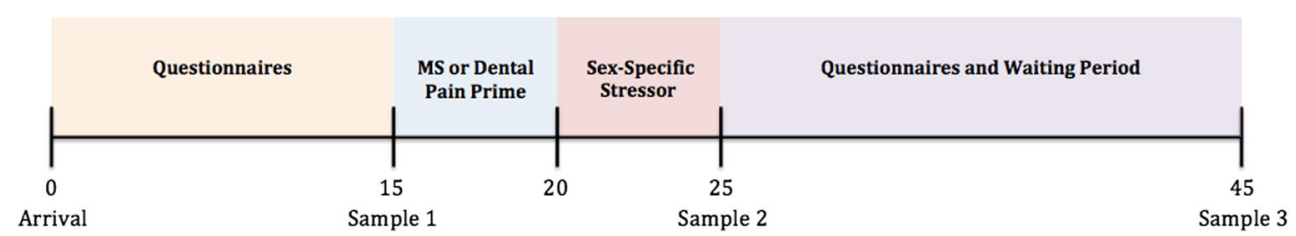

assessing their desire to affiliate with others, including close others (e.g., family, friends, partners) and their fellow participants.

\section{Data Analysis}

To determine the change in hormonal reactivity to the stressors, a three-way ANOVA was computed using mortality salience (MS) condition (MS or dental prime), video stressor (out-group threat or crying babies), and sex as the betweenparticipant individual variables, and hormonal (cortisol or testosterone) change scores as the dependent variable. The cortisol and testosterone change scores were computed to determine the change in reactivity from immediately after the stressor (initial reaction) to $20 \mathrm{~min}$ post-stressor (peak reaction and beginning of recovery period). This method of estimating reactivity was chosen over other method (e.g., area under the curve or AUC) due to the relatively limited number of samples collected over a brief period. In studies with less than four data collection points, AUC is unlikely to yield information over and above change scores (Pruessner et al. 2003).

Next, we used a categorical approach to better understand response patterns to the stimuli. This was done using two criterion that allow us to infer that the magnitude of the difference observed between the two time points is larger than measurement error per Granger et al. (2012) These two criterion are (1) at least a $10 \%$ difference between hormone concentrations and (2) an absolute difference between the scores greater than $.02 \mu \mathrm{g} / \mathrm{dL}$, which corresponds to at least twice the average intra-assay coefficient of variation (CV). Thus, testosterone and cortisol concentrations were converted into percent changes by subtracting the T3 concentrations from T2, and then dividing that change value by the $\mathrm{T} 2$ concentration value and converted into a percentage value (Allwood et al. 2011). All analyses are subsequently described in percent change results and ANOVAs were conducted only on those who were "responders," i.e., greater than a $10 \%$ increase in either marker (Glenn et al. 2011). See Table 1 for the pattern of responders and "non-responders." In order to test hypotheses, a $2 \times 2 \times 2$ between-subjects ANOVA was first conducted using mortality salience group (dental pain control prime or MS), social stressor (Westboro Baptist Church or crying babies), and sex on percent changes from T2 to T3 of cortisol and testosterone.

\section{Results}

Cortisol There were no significant results in cortisol reactivity from times 1 to 2 . This was not unanticipated, as cortisol typically takes 15-20 min post-stressor to peak (Gordis et al. 2006). As predicted, there was a main effect of condition for cortisol reactivity (times 2 to 3 ), $F(1151)=3.31, p=.02$. Follow-up comparisons revealed that there were significant differences in cortisol reactivity to the control/crying babies condition and the control/out-group threat condition $(p=.01)$, with reactivity being stronger to the out-group threat condition. Further, the MS/crying babies group significantly differed from the control/out-group threat condition, with the out-group threat condition again generating a stronger cortisol response $(p=.009)$.

Of the cortisol responders, females showed significantly higher self-reported desire to be with close others (e.g., friends, family) following the stressor regardless of morality salience prime or social stressor experienced, $F(1,56)=5.78$, $p=.02$, partial $\eta^{2}=.09$. Social stressor showed a significant main effect, $F(1,56)=7.06, p=.01$, partial $\eta^{2}=.11$, whereas sex and mortality salience were not significant predictors of cortisol percent changes, $p s=.45$ and .23 , respectively. None of the two-way interaction terms were significant, $p \mathrm{~s}>.15$; however, the three-way interaction was significant, $F(1$, $56)=4.37, p=.04$, partial $\eta^{2}=.07$. See Figs. 2 and 3 .

Table 1 Total sample numbers, "non-responders," and "responders" by experimental condition

\begin{tabular}{|c|c|c|c|c|c|c|c|c|}
\hline \multicolumn{4}{|c|}{ Cortisol } & \multirow[t]{2}{*}{ Condition } & \multicolumn{4}{|c|}{ Testosterone } \\
\hline Total & Non-responders & Responders & $\%$ Responders & & Total & Non-responders & Responders & \% Responders \\
\hline 46 & 23 & 23 & 50 & Control/babies & 46 & 35 & 11 & 23 \\
\hline 39 & 24 & 14 & 36 & Control/out-group threat & 39 & 29 & 8 & 20 \\
\hline 19 & 10 & 9 & 47 & MS/babies & 19 & 14 & 5 & 26 \\
\hline 49 & 30 & 9 & 18 & MS/out-group threat & 49 & 38 & 11 & 22 \\
\hline
\end{tabular}




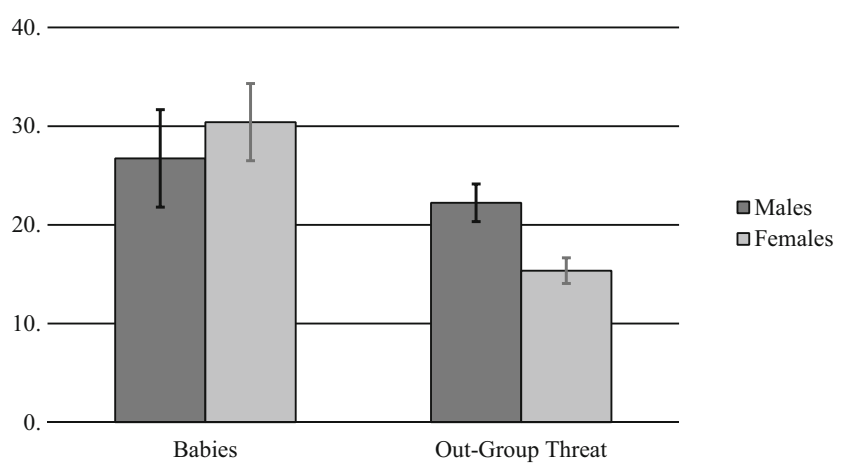

Fig. 2 Mortality salience condition: cortisol percent changes from T2 to $\mathrm{T} 3$

To better understand the simple main effects and interactions, a series of significance tests of the simple slopes for the three-way interaction was conducted using a Bonferroni correction. In order to test the experimental manipulation, males, the MS control group, and the crying babies condition were used as reference groups for sex, the experimental groups, and the video stressor, respectively. There was no significant difference between the MS control and the MS groups when viewing the out-group threat stressor $(p=.85)$; however, there was a marginally significantly higher percentage change in participants who viewed the crying babies who were in the MS group, $F(1,56)=3.38, p=.07$, partial $\eta^{2}=.06$. A marginal significant difference was found between males and females in the MS control condition $(p=.10)$, but no significant difference between males and females in the MS condition. There was a significant difference between the crying babies and out-group threat in females in the mortality salience condition, with the crying babies condition showing significantly higher cortisol change $(p=.01)$.

Testosterone As with cortisol, there were no significant results in testosterone reactivity from times 1 to 2 , though this was not unexpected. There was a marginally significant main effect of sex for testosterone reactivity (times 2 to 3 ), $F(1151)=3.03, p=.07$. However, there were no other significant main effects, nor were there significant interactions between sex and experimental condition.

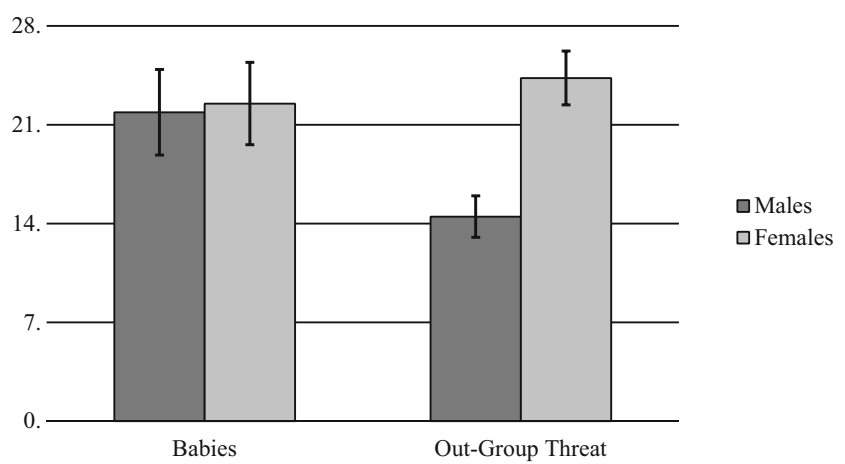

Fig. 3 MS control condition: cortisol percent changes from T2 to T3
With respect to the testosterone responders, in the initial three-way ANOVA, the mortality salience condition was not a significant predictor $(p=.66)$, nor were its interactions with sex $(p=.20)$ or social stressor $(p=.53)$; therefore, the model was condensed to a 2 (sex) $\times 2$ (social stressor) betweensubjects ANOVA ( $N=35,17$ males). Neither main effect was significant $(p s>.63)$. The interaction effect was significant. Males had a higher average testosterone response to the out-group threat, and females had a higher average percentage change in testosterone response to the crying babies, with a significant interaction, $F(1,31)=6.96, p=.01$, partial $\eta^{2}=.18$. See Fig. 4.

\section{Discussion}

Results add an additional layer of complexity to the physiological cascades that occur when examining male- and femaletypical social stressors previously unaccounted for in tendand-befriend models. This study demonstrated that males and females are sensitive to and show a different pattern of physiological responses to somewhat different social stimuli. Perhaps most novel, and consistent with our hypothesis, the results showed a female testosterone response to cues of infant distress. This is consistent with a hypothesized hormonal sequence in which testosterone relates to the production of estradiol, related to many caretaking behaviors (WynneEdwards and Reburn 2000). Some researchers posit that increases in testosterone related to infants and social behavior are due to the contribution of testosterone in the synthesis of estradiol (e.g., Bos et al. 2012). Thus, the increases in testosterone in the current study in response to the infant crying condition may be due to the facilitation of estradiol production. This sequence of hormonal events is, however, unaccounted for in traditional tend-and-befriend models of responding in a sex-specific manner.

The male testosterone response to the out-group threat is consistent with the traditional Challenge Hypothesis (Wingfield et al. 1990) in which a testosterone response is mounted in a presumed attempt to meet the demands of a

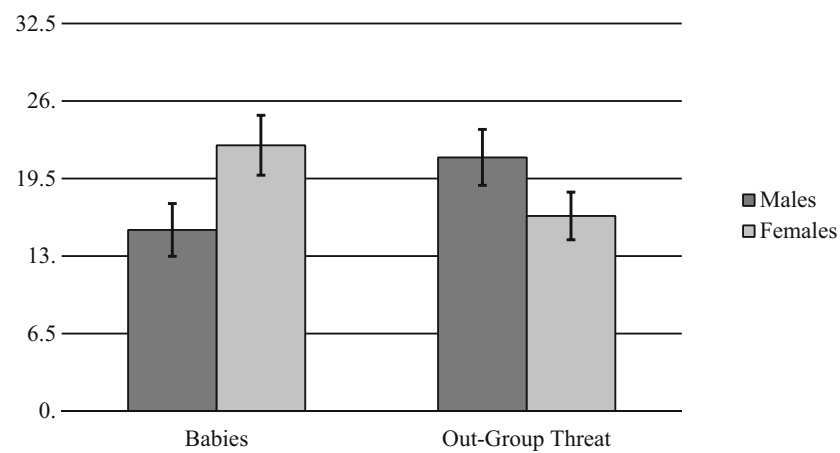

Fig. 4 Testosterone percent changes from T2 to T3 
potential threat to one's coalition. Corresponding testosterone responses and competitive motivation has been shown to differ for males, particularly when competing in coalitions or teams (Kivlighan et al. 2005; Mazur et al. 1992). Our results show that even relatively remote and vague threats to one's coalition, such as those experienced in the out-group threat condition in this study, are capable of mounting a testosterone response. This is also consistent with evolutionary models suggesting that males have evolved biases that enable the formation of cohesive in-groups aimed at competing against out-group threats (Geary et al. 2003).

It is important to note that testosterone responses, for both males and females, appeared to not be influenced by mortality salience primes. This may demonstrate the relative independence of the two systems (HPG and HPA) in responding to social stressors, in that cortisol responses were dependent on mortality salience condition, particularly for males. The HPA axis is particularly responsive to psychosocial contexts signaling threat, risk, opportunity, and/or uncontrollable social dynamics (Dickerson and Kemeny 2004; Flinn et al. 2012; Hellhammer et al. 2009), and mortality salience may represent one such context.

The cortisol results are consistent with previous research (Byrd-Craven et al. 2015). These results lend support to Geary and Flinn's (2002) extension of tend-and-befriend by showing that males are responsive to cues of out-group threat. The data suggest that mortality cues influence sex-specific physiological cascades of responses. Males who did not experience mortality cues showed a lower cortisol response to cues of outgroup threat. In combination with the testosterone responses to out-group threat, this pattern of responding is consistent with a response that may behaviorally prepare men to respond to a threat. Suppressed adrenal activity modulated by testosterone has been consistently demonstrated in animal studies (Bingaman et al. 1994; Burgess and Handa 1992; Critchlow et al. 1963; Handa et al. 1994). Specific to men's responses to out-group threat, the pattern of lower cortisol and higher testosterone response has been demonstrated in both naturalistic and simulated studies of out-group competition (Flinn et al. 2012; Oxford et al. 2010).

Cortisol and testosterone are two factors in a sequence of hormonal mechanisms that regulate other hormonal and behavioral responses to social threats and challenges. Both sexes exhibit a rapid cortisol and testosterone response to a range of social challenges. To our knowledge, this is one of the first of studies to investigate the mechanisms that underlie Geary and Flinn (2002) suggested modifications to the Tend-and-Befriend model. Limitations include a relatively small sample size and lack of control for ovulatory status in women. The timing of the menstrual cycles influences cortisol and testosterone responses, as well as how women are influenced by mortality salience cues (Vaughn et al. 2010). It is plausible that menstrual timing may have influenced responses to both social stressors. Even given these limitations, however, the current study provides unique and valuable information regarding the sex-specific pattern of hormonal responses to social stimuli. Consistent with previous research, females showed a cortisol response to infant distress. The testosterone response to infant distress is more novel and provides avenues for future investigation of the cascade of hormonal events relevant to the tending response. Further, mortality cues appear to be more salient to the sequence of hormone responses for men, and may be more important to men's coalitional behavior compared with women's responses to infant distress.

\section{References}

Allwood, M. A., Handwerger, K., Kivlighan, K. T., Granger, D. A., \& Stroud, L. R. (2011). Direct and moderating links of salivary alphaamylase and cortisol stress-reactivity to youth behavioral and emotional adjustment. Biologicaly Psychology, 88(1), 57-64. doi:10. 1016/j.biopsycho.2011.06.008.

Arndt, J., Greenberg, J., \& Cook, A. (2002). Mortality salience and the spreading activation of worldview-relevant constructs: exploring the cognitive architecture of terror management. Journal of Experimental Psychology-General, 131(3), 307-324. doi:10.1037// 0096-3445.131.3.307.

Bernhardt, P. C., Dabbs, J. M., Fielden, J. A., \& Lutter, C. D. (1998). Testosterone changes during vicarious experiences of winning and losing among fans at sporting events. Physiology \& Behavior, 65(1), 59-62. doi:10.1016/s0031-9384(98)00147-4.

Bingaman, E. W., Vandekar, L. D., Yracheta, J. M., Li, Q., \& Gray, T. S. (1994). Androgens differentially influence neuroendocrine and behavioral responses to psychological stress in the rat. FASEB Journal, 8(4), A110-A110.

Bos, P. A., Hermans, E. J., Montoya, E. R., Ramsey, N. F., \& van Honk, J. (2010). Testosterone administration modulates neural responses to crying infants in young females. Psychoneuroendocrinology, 35(1), 114-121. doi:10.1016/j.psyneuen.2009.09.013.

Bos, P. A., Panksepp, J., Bluthé, R.-M., \& Honk, J. V. (2012). Acute effects of steroid hormones and neuropeptides on human socialemotional behavior: a review of single administration studies. Frontiers in Neuroendocrinology, 33(1), 17-35. doi:10.1016/j. yfrne.2011.01.002.

Burgess, L. H., \& Handa, R. J. (1992). Chronic estrogen-induced alterations in adrenocorticotropin and corticosterone secretion, and glucocorticoid receptor-mediated functions in female rats. Endocrinology, 131(3), 1261-1269. doi:10.1210/en.131.3. 1261.

Byrd-Craven, J., Auer, B. J., \& Kennison, S. M. (2015). Sex differences in salivary cortisol responses to sex-linked stressors: a test of the tend-and-befriend model. Adaptive Human Behavior and Physiology, 1(4), 408-420. doi:10.1007/s40750-014-0013-1.

Cobey, K. D., Klipping, C., \& Buunk, A. P. (2013). Hormonal contraceptive use lowers female intrasexual competition in pair-bonded women. Evolution and Human Behavior, 34(4), 294-298. doi:10.1016/j. evolhumbehav.2013.04.003.

Critchlow, V., Barsela, M., Mountcastle, W., Liebelt, R. A., \& Lipscomb, H. S. (1963). Sex difference in resting pituitary-adrenal function in the rat. American Journal of Physiology, 205(5), 807-815. 
Dickerson, S. S., \& Kemeny, M. E. (2004). Acute stressors and cortisol responses: a theoretical integration and synthesis of laboratory research. Psychological Bulletin, 130(3), 355-391. doi:10.1037/00332909.130.3.355.

Fleming, A. S., Corter, C., Stallings, J., \& Steiner, M. (2002). Testosterone and prolactin are associated with emotional responses to infant cries in new fathers. Hormones and Behavior, 42(4), 399 413. doi:10.1006/hbeh.2002.1840.

Fleming, A. S., Ruble, D., Krieger, H., \& Wong, P. Y. (1997a). Hormonal and experiential correlates of maternal responsiveness during pregnancy and the puerperium in human mothers. Hormones and Behavior, 31(2), 145-158. doi:10.1006/hbeh. 1997.1376.

Fleming, A. S., Steiner, M., \& Corter, C. (1997b). Cortisol, hedonics, and maternal responsiveness in human mothers. Hormones and Behavior, 32(2), 85-98. doi:10.1006/hbeh.1997.1407.

Flinn, M. V., Ponzi, D., \& Muehlenbein, M. P. (2012). Hormonal mechanisms for regulation of aggression in human coalitions. Human nature - an interdisciplinary biosocial perspective, 23(1), 68-88. doi:10.1007/s12110-012-9135-y.

Geary, D. C. (2010). Male, female: the evolution of human sex differences. Washington, DC: American Psychological Association

Geary, D. C., Byrd-Craven, J., Hoard, M. K., Vigil, J., \& Numtee, C. (2003). Evolution and development of boys' social behavior. Developmental Review, 23(4), 444-470.

Geary, D. C., \& Flinn, M. V. (2002). Sex differences in behavioral and hormonal response to social threat: commentary on Taylor et al. (2000). Psychological Review, 109(4), 745.

Giardino, J., Gonzalez, A., Steiner, M., \& Fleming, A. S. (2008). Effects of motherhood on physiological and subjective responses to infant cries in teenage mothers: a comparison with non-mothers and adult mothers. Hormones and Behavior, 53(1), 149-158. doi:10.1016/j. yhbeh.2007.09.010.

Glenn, A. L., Raine, A., Schug, R. A., Gao, Y., \& Granger, D. A. (2011). Increased testosterone to cortisol ratio in psychopathy. Journal of Abnormal Psychology, 120(2), 389-399. doi:10. 1037/a0021407.

Gonzalez-Bono, E., Salvador, A., Ricarte, J., Serrano, M. A., \& Arnedo, M. (2000). Testosterone and attribution of successful competition. Aggressive Behavior, 26(3), 235-240. doi:10.1002/(sici)10982337(2000)26:3<235::aid-ab3>3.0.co;2-1.

Gordis, E. B., Granger, D. A., Susman, E. J., \& Trickett, P. K. (2006). Asymmetry betweenc salivary cortisol and $\alpha$-amylase reactivity to stress: Relation to aggressive behavior in adolescents. Psychoneuroendocrinology, 31, 976-987. doi:10.1016/j.psyneuen. 2006.05.010

Granger, D. A., Fortunato, C. K., Beltzer, E. K., Virag, M., Bright, M. A., \& Out, D. (2012). Focus on methodology: salivary bioscience and research on adolescence: an integrated perspective. Journal of Adolescence, 35, 1081-1095.

Greenberg, J., Pyszczynski, T., \& Solomon, S. (1986). The causes and consequences of a need for self-esteem: a terror management theory. In Public self and private self (pp. 189-212). Springer New York.

Handa, R. J., Nunley, K. M., Lorens, S. A., Louie, J. P., McGivern, R. F., \& Bollnow, M. R. (1994). Androgen regulation of adrenocorticotropin and corticosterone secretion in the male rat following novelty and foot shock stressors. Physiology \& Behavior, 55, 117-124. doi: 10.1016/0031-9384(94)90018-3.

Hellhammer, D. H., Wuest, S., \& Kudielka, B. M. (2009). Salivary cortisol as a biomarker in stress research. Psychoneuroendocrinology, 34(2), 163-171. doi:10.1016/j.psyneuen.2008.10.026.

Hrdy, S. B. (2007). Evolutionary context of human development: the cooperative breeding model. In C. A. Salmon \& T. K. Shackelford
(Eds.), Family relationships: an evolutionary perspective (pp. 3968). New York: Oxford University Press.

Kivlighan, K. T., Granger, D. A., \& Booth, A. (2005). Gender differences in testosterone and cortisol response to competition. Psychoneuroendocrinology, 30(1), 58-71.

Kurzban, R., Tooby, J., \& Cosmides, L. (2001). Can race be erased? Coalitional competition and social categorization. Proceeding of the National Academy of Sciences, 98, 15387-15392.

Maner, J. K., \& McNulty, J. K. (2013). Attunement to the fertility status of same-sex rivals: women's testosterone responses to olfactory ovulation cues. Evolution and Human Behavior, 34(6), 412-418. doi:10. 1016/j.evolhumbehav.2013.07.005.

Mazur, A., Booth, A., \& Dabbs, J. M. (1992). Testosterone and chess competition. Social Psychology Quarterly, 55(1), 70-77. doi:10. 2307/2786687.

Miller, S. L., Maner, J. K., \& McNulty, J. K. (2012). Adaptive attunement to the sex of individuals at a competition: the ratio of opposite- to same-sex individuals correlates with changes in competitors' testosterone levels. Evolution and Human Behavior, 33(1), 57-63. doi:10. 1016/j.evolhumbehav.2011.05.006.

Oxford, J., Ponzi, D., \& Geary, D. C. (2010). Hormonal responses differ when playing violent video games against an ingroup and outgroup. Evolution and Human Behavior, 31(3), 201-209. doi:10.1016/j. evolhumbehav.2009.07.002.

Pruessner, J., Kirschbaum, C., Meinlschmid, G., \& Hellhammer, D.H. (2003). Two formulas for computation of the area under the curve represent measures of total hormone concentration versus time-dependent change. Psychoneuroendocrinology, 28, 916-931.

Savin-Williams, R. C. (1979). Dominance hierarchies in groups of early adolescents. Child Development, 50(4), 923. doi:10.1111/14678624.ep7251679.

Solomon, S., Greenberg, J., \& Pyszczynski, T. (1991). A terror management theory of social behavior: the psychological functions of self-esteem and cultural worldview. Advances in Experimental Social Psychology, 24, 93-159. doi:10.1016/ s0065-2601(08)60328-7.

Stallings, J. F., Fleming, A. S., Worthman, C. M., Steiner, M., Corter, C., \& Coote, M. (1997). Mother/father differences in response to infant crying. American Journal of Physical Anthropology, (SUPPL. 24), 217-217.

Storey, A. E., Walsh, C. J., Quinton, R. L., \& Wynne-Edwards, K. E. (2000). Hormonal correlates of paternal responsiveness in new and expectant fathers. Evolution and Human Behavior, 21(2), 79-95. doi:10.1016/s1090-5138(99)00042-2.

Taylor, S. E. (2006). Tend and befriend: biobehavioral bases of affiliation under stress. Current Directions in Psychological Science, 15(6), 273-277. doi:10.1111/j.1467-8721.2006.00451.x.

Taylor, S. E., Klein, L. C., Lewis, B. P., Gruenewald, T. L., Gurung, R. A. R., \& Updegraff, J. A. (2000). Biobehavioral responses to stress in females: tend-and-befriend, not fight-or-flight. Psychological Review, 107(3), 411-429. doi:10.1037/0033295X.107.3.411.

van Anders, S. M. (2013). Beyond masculinity: testosterone, gender/sex, and human social behavior in a comparative context. Frontiers in Neuroendocrinology, 34(3), 198-210. doi:10.1016/j.yfrne.2013.07. 001.

van Anders, S. M., Goldey, K. L., \& Kuo, P. X. (2011). The steroid/ peptide theory of social bonds: integrating testosterone and peptide responses for classifying social behavioral contexts. Psychoneuroendocrinology, 36(9), 1265-1275. doi:10.1016/j. psyneuen.2011.06.001.

van Anders, S. M., Tolman, R. M., \& Volling, B. L. (2012). Baby cries and nurturance affect testosterone in men. Hormones and Behavior 61(1), 31-36. doi:10.1016/j.yhbeh.2011.09.012. 
Vaughn, J. E., Bradley, K. I., Byrd-Craven, J., \& Kennison, S. M. (2010). The effect of mortality salience on women's judgments of male faces. Evolutionary Psychology, 8(3), 477-491.

Wagner, J. D., Flinn, M. V., \& England, B. G. (2002). Hormonal response to competition among male coalitions. Evolution and Human Behavior, 23(6), 437-442. doi:10.1016/s1090-5138(02) 00100-9.

Wingfield, J. C., Hegner, R. E., Dufty, A. M., \& Ball, G. F. (1990). The "challenge hypothesis": theoretical implications for patterns of testosterone secretion, mating systems, and breeding strategies. American Naturalist, 136(6), 829-846. doi:10.1086/285134.

Wisman, A., \& Koole, S. L. (2003). Hiding in the crowd: can mortality salience promote affiliation with others who oppose one's worldviews? Journal of Personality and Social Psychology, 84(3), 511526. doi:10.1037/0022-3514.84.3.511.

Wynne-Edwards, K. E., \& Reburn, C. J. (2000). Behavioral endocrinology of mammalian fatherhood. Trends in Ecology \& Evolution, 15(11), 464-468. doi:10.1016/s0169-5347(00) 01972-8. 